\title{
THE EFFECTS OF ADRENALINE, NORADRENALINE, AND ISOPRENALINE ON PARKINSONIAN TREMOR
}

\author{
C. CONSTAS \\ From the Academic Unit, Institute of Neurology, and National Hospitals for Nervous Diseases, \\ Queen Square, London
}

The development of pharmacological substances, capable of exerting powerful effects at known sites in the nervous system, has provided a new versatile tool for clinical research. No longer is it necessary to destroy a part of the nervous system in order to study what effect it may have upon a phenomenon; the activity of that part may be held in abeyance by the use of pharmacological blocking agents. Likewise, stimulation no longer always demands the application of electrodes; drugs acting at a specific site provide a more elegant experiment. But the supreme advantage is that these methods can be applied to man, thus eliminating the many pitfalls which arise when human problems are studied in other species.

The aggravation of Parkinsonian tremor which occurs when a patient becomes emotionally upset has long been attributed to the release of adrenaline, and Barcroft, Peterson, and Schwab (1952) have shown that the infusion of adrenaline into patients suffering from Parkinsonism increases their tremor. These workers had not, however, the means at their disposal of ascertaining the site of action of the adrenaline. There is evidence that adrenaline can act at several sites in the nervous system. Thus, Marazzi (1960) has shown that adrenaline may cause inhibition at cortical synapses, and McLennan (1961) found that catechol amines, when applied to the exposed spinal cord of the cat, inhibit the knee jerk. The present study was carried out to ascertain the site at which catechol amines act in aggravating Parkinsonian tremor.

\section{METHOD}

Tremors were recorded by means of a valve, sensitive to acceleration, which the patient, while sitting comfortably in a chair, held in the outstretched hand. The output of the valve upset the balance of a bridge circuit and after amplification was recorded by a pen-writer on moving paper. The apparatus was fully described by Marshall and Walsh (1956). In all the experiments the gain remained constant.

Twenty-five patients were studied, 15 males and 10 females, with an age range of 38 to 56 years. All were suffering from unmistakable Parkinsonism, no attempt being made to distinguish the aetiological cause. They showed no clinical evidence of cerebrovascular or cardiovascular disease and their blood pressures ranged from 120 to $155 \mathrm{~mm}$. Hg systolic to 70 to $95 \mathrm{~mm}$. diastolic.

\section{TYPES OF EXPERIMENTS AND RESULTS}

THE EFFECT OF ADRENALINE One millilitre of a $1 / 1,000$ solution of adrenaline B.P. was injected intramuscularly in each of the 25 patients. This produced a feeling of excitement, apprehension, or uneasiness, accompanied by palpitation, pallor of the skin, and an increase in the pulse rate of 25 to $30 \%$. The systolic blood pressure was increased by $25 \%$. The diastolic pressure was decreased by $20 \%$ in 10 patients, increased by $25 \%$ in 10 , and unchanged in five.

The amplitude of the tremor was greatly increased in all the patients except one (Fig. 1). The increase became evident three to five minutes after the injection, reaching a peak in eight to 10 minutes and persisting for 20 to 25 minutes. The degree of increase in the amplitude is shown in Table I.

TABLE I

INCREASE IN AMPLITUDE OF TREMOR AFTER INTRAMUSCULAR ADRENALINE

\begin{tabular}{cccc}
$\begin{array}{l}\text { Percentage Increase } \\
\text { in Amplitude }\end{array}$ & Males & Females & Total \\
\hline 50 & 3 & -1 & 3 \\
75 & 3 & - & 4 \\
100 & 3 & 2 & 3 \\
150 & 1 & 3 & 3 \\
200 & 2 & 4 & 5 \\
$250+$ & 3 & 10 & 7 \\
Total & 15 & & 25
\end{tabular}

The increase in amplitude was more evident in women than in men. The frequency of the tremor was also increased in eight of the patients (four males and four females). This was measured by placing a transparent cursor over the record and counting the number of cycles in a series of samples 
a

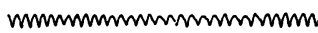

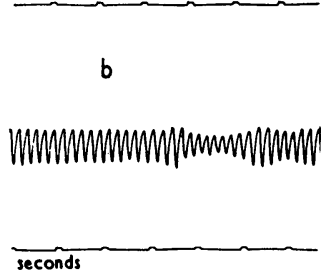

FIG. 1

a

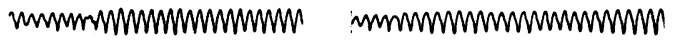

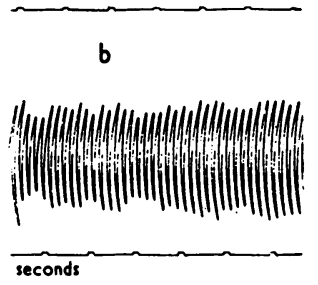

FIG. 2

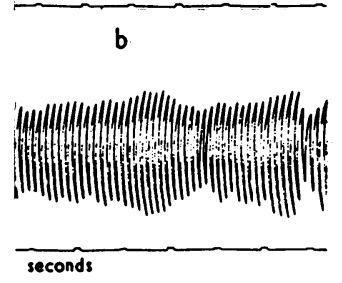

FIG. 3

FIG. 1. a Tremor recorded before the administration of adrenaline. $b$ After the intramuscular administration of $1 \mathrm{mg}$. of adrenaline (increase in amplitude).

FIG. 2. a Before the administration of adrenaline. $b$ After the intramuscular administration of adrenaline (increase in amplitude and frequency).

FIG. 3. $a$ Before the administration of adrenaline. $b$ After the intravenous infusion of adrenaline.

each of $1 \mathrm{sec}$. duration. The resting tremor of the above patients was 4 to $6 \mathrm{c} / \mathrm{s}$. In four (three males and one female) the frequency was increased by 1 to $2 \mathrm{c} / \mathrm{s}$, in three (one male and two females) by 2 to $3 \mathrm{c} / \mathrm{s}$, and in one (female) by 3 to $4 \mathrm{c} / \mathrm{s}$ (Fig. 2). Again in women there was a tendency for a greater increase in the frequency of the tremor than in men.

In three patients (two males and one female) adrenaline, diluted in isotonic saline, was given by intravenous infusion at a rate of $20 \mu \mathrm{g}$. per minute. The increase of tremor was observed 45 seconds after the beginning of the infusion and there was no significant difference in the increase in the amplitude of the tremor from that observed after intramuscular administration (Fig. 3).

THE EFFECT OF SALINE In order to exclude the possibility that apprehension of the experimental situation was causing the increase in amplitude of the tremor, the injection of adrenaline was preceded by an intramuscular injection of sterile normal saline in 10 of the patients. This produced no change in the amplitude or frequency of the tremor (Fig. 4), except in one instance where there was a negligible increase in amplitude of less than $20 \%$.

EFFECT OF PRECEDING MEDICATION Five patients were given anti-Parkinsonian drugs before the administration of adrenaline. They received Artane, $2 \mathrm{mg}$. q.d.s., for the preceding 24 hours. The increase in the amplitude of the tremor caused by the adrenaline was less than it had been when recorded without premedication (Table II and Fig. 5). The general symptoms were unaffected. a

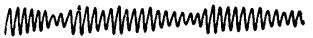
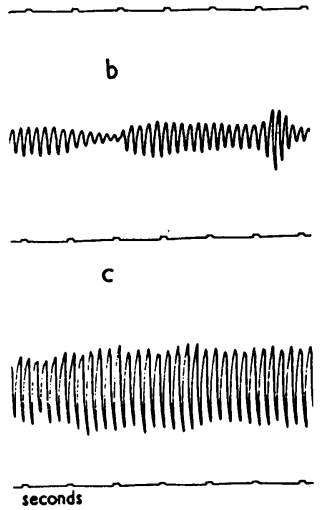

FIG. 4 o

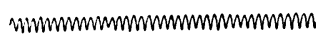

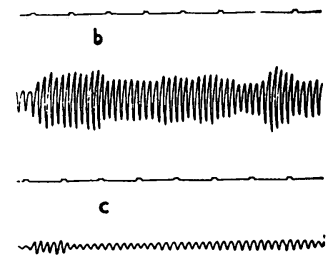

d

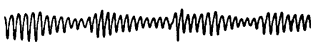

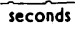

FIG. 5
FIG. 4. a Tremor recorded before administration of saline.

$b$ After the intramuscular administration of saline.

c After the intramuscular administration of adrenaline.

FIG. 5. The action of adrenaline before and after the administration of anti-Parkinsonian drugs.

a Before any medication.

$b$ After the administration of adrenaline.

$c$ After the administration of Artane, 2 mg. q.d.s.

$d$ After the administration of adrenaline on the patient receiving Artane. 
TABLE II

\begin{tabular}{cccc}
\multicolumn{3}{c}{ PERCENTAGE CHANGE IN AMPLITUDE OF TREMOR } \\
Patient & $\begin{array}{l}\text { Adrenaline } \\
\text { (1 mg. intra- } \\
\text { muscularly) }\end{array}$ & $\begin{array}{l}\text { Artane } \\
\text { (2 mg. q.d.s.) }\end{array}$ & $\begin{array}{l}\text { Artane }(2 \text { mg.q.d.s. }) \\
\text { Followed by } \\
\text { Adrenaline }(1 \text { mg. } \\
\text { intramuscularly) }\end{array}$ \\
\hline 1 & +100 & -50 & +75 \\
2 & +150 & -25 & +100 \\
3 & +75 & -25 & +75 \\
4 & +300 & -50 & +200 \\
5 & +300 & -50 & +200
\end{tabular}

THE ACTION OF NORADRENALINE Isotonic saline was first administered by intravenous infusion at the rate of $2 \mathrm{ml}$. per minute for five minutes during which time the blood pressure and pulse rate were measured. Noradrenaline was then infused at a rate of 5 to $30 \mu \mathrm{g}$. per minute. With small doses $(10 \mu \mathrm{g}$. per min.) none of the patients had any complaints, except for one who noted a feeling of constriction in the chest. With larger doses $(30 \mu \mathrm{g}$. per min.) all patients experienced a feeling of tightness in the chest and mild palpitations. About 45 seconds after the start of the infusion a definite blanching of the face, lips, and mucosa of the mouth was observed. This persisted throughout the infusion. A less definite pallor was seen in the limbs. The infusion was continued for 10 to 15 minutes and when it was stopped the patients complained of a feeling of warmth in the face and limbs lasting for a few minutes. No patient experienced any symptoms of anxiety. The systolic blood pressure of the patients was increased by $\mathbf{3 0}$ to $50 \%$. There was no significant change in the amplitude or frequency of the tremor (Fig. 6). After the infusion of noradrenaline, adrenaline was given

o

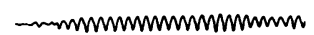

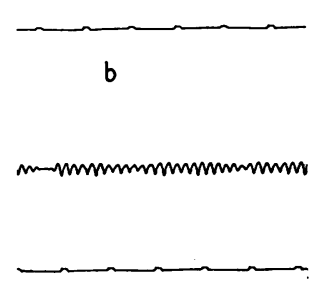

FIG. 6.

The action of noradrenaline. a Before the administration of noradrenaline.

$b$ After the intravenous infusion of noradrenaline. $c$ After the intravenous infusion of adrenaline.

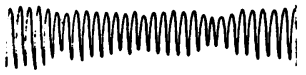

seconds $\mathbf{0}$

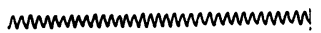

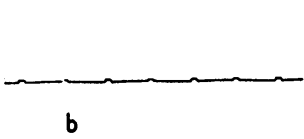

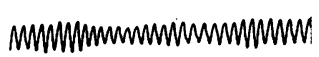

FIG. 7

The action of isoprenaline. $a$ Before the administration of isoprenaline.

$b$ After the administration of isoprenaline (increase in amplitude).

seconds

by the same route at the same rate, with marked increase in the amplitude of tremor.

THE ACTION OF ISOPRENALINE Neo-epininine (Burroughs Wellcome) containing $1 \%$ of isoprenaline sulphate was administered by oral inhalation in a dose of $0.3 \mathrm{ml}$. to five patients. Two of the patients complained of mild uneasiness and apprehension and one of palpitations. The systolic pressure increased by $20 \%$ in two patients and the pulse rate by $22 \%$. No significant change was noted in the diastolic pressure. There was no significant change in the tremor in two male patients. In two other patients (one male and one female) the tremor was increased in amplitude by 30 to $70 \%$ (Fig. 7). In the fifth patient (a woman) the amplitude was increased by $30 \%$ and the frequency by 1 to $2 \mathrm{c} / \mathrm{s}$. The effect on the tremor lasted five minutes after the inhalation was stopped.

SITE OF ACTION OF ADRENALINE A series of experiments was then carried out to ascertain the site of action at which the adrenaline caused the increase in the tremor.

Adrenaline was administered in five patients into the brachial artery proximal to the elbow over the point of the maximal pulsation in the antecubital fossa. A sphygmomanometer cuff inflated to above diastolic blood pressure was in position above the site of injection.

In one subject adrenaline was infused at a rate of $10 \mu \mathrm{g}$. per minute. In four subjects adrenaline was administered by an acute intra-arterial injection in a dose of 0.1 to $0.3 \mathrm{mg}$. Within a minute marked pallor was observed starting in the fingers and spreading to the hand, and once up to the elbow. This was particularly marked with larger amounts (more than $0.1 \mathrm{mg}$.). Marked coldness was noticed also. The radial pulse became smaller whereas the pulse in the other arm remained the same. The local reaction reached its peak in two to three minutes and then gradually subsided over the next 10 minutes. With doses up to $0.1 \mathrm{mg}$. no change in the pulse or 
blood pressure was observed, but with larger doses $(0.2$ to $0.3 \mathrm{mg}$.) there was a little increase in the blood pressure and the pulse rate. In no case was there any increase in the tremor. There was, however, a slight increase in amplitude after the cuff of the sphygmomanometer was released. An intramuscular injection of $0.3 \mathrm{mg}$. adrenaline in these patients produced the expected increase in the amplitude and frequency of the tremor (Fig. 8).

ACTION OF ADRENALINE AFTER ADMINISTRATION OF GANGLION-BLOCKING DRUGS (HEXAMETHONIUM) In five patients $1 \mathrm{mg}$. of adrenaline was injected intramuscularly and the usual increase in the tremor was observed. Forty-five minutes after the injection, by which time the tremor had reverted to normal, an intravenous infusion of normal saline was begun with the patient lying comfortably. Hexamethonium iodide was injected into the infusion needle in doses of $5 \mathrm{mg}$. until the blood pressure fell. Usually doses

$\circ$

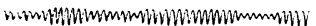

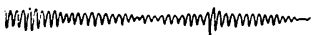

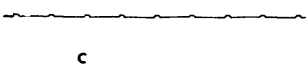

It:

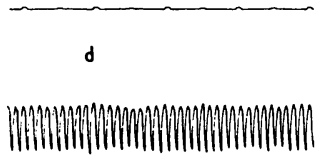

seconds

FIG. 8

of 20 to $25 \mathrm{mg}$. were adequate to produce a fall in pressure of 20 to $25 \%$ systolic and 10 to $15 \%$ diastolic. A further injection of adrenaline ( $1 \mathrm{mg}$.) was then given intramuscularly.

After the second administration of adrenaline the blood pressure rose to the previous levels, and then increased by a further $15 \%$. There was no significant difference in the increase of the adrenaline-induced tremor before and after the administration of the hexamethonium (Fig. 9).

ACTION OF ADRENALINE AFTER ADMINISTRATION OF PHENTOLAMINE (ROGITINE) In order to ascertain more closely the site of action of adrenaline, phentolamine was employed. This interferes with the response of the effector organs to the excitatory actions of adrenaline by creating a competitive block at the end synapse of the postganglionic fibre. In seven patients adrenaline was given intramuscularly in doses of $1 \mathrm{mg}$. and the tremor again

$\mathbf{a}$

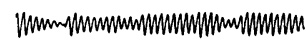
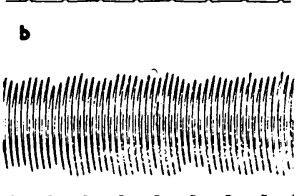

c
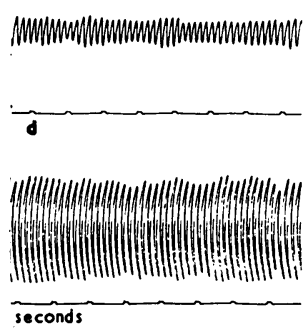

FIG. 10
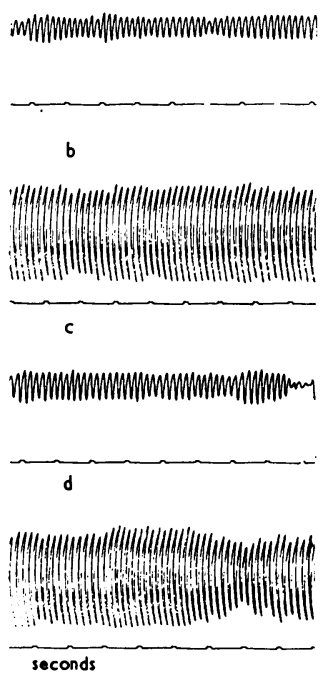

FIG. 11

FIG. 8. The action of intra-arterially administered adrenaline. a Before the administration of adrenaline. $b$ After the administration of adrenaline. $c$ After the release of the cuff. $d$ The same amount of adrenaline given intramuscularlv.

FIG. 9. The action of adrenaline on the tremor before and after the administration of hexamethonium. a Patient received no premedication. $b$ After the administration of adrenaline. $c$ After the administration of hexamethonium, 45 min. after the first experiment. d After the administration of adrenaline (no difference in the action of the adrenaline).

FIG. 10. The action of adrenaline before and after the administration of phentolamine. a Patient receiving no medication. $b$ After the administration of adrenaline. $c$ After the intravenous administration of phentolamine (45 min. after the first experiment). $d$ After the administration of adrenaline (no difference in the action of the adrenaline).

FIG. 11. The action of adrenaline on the tremor before and after the administration of guanethidine. a Patient receiving no medication. $b$ After the administration of adrenaline. $c$ Patient receiving guanethidine. $d$ After the administration of adrenaline (no difference in the action of the adrenaline). 
recorded. Forty-five minutes later phentolamine was given intravenously in doses varying from 5 to $15 \mathrm{mg}$. The injection of adrenaline was repeated intramuscularly at the same dosage $(1 \mathrm{mg}$.) five to 10 minutes after the phentolamine injection. There was no change in the adrenaline-induced tremor before and after the phentolamine (Fig. 10).

EFFECT OF ADRENALINE BEFORE AND AFTER ADMINISTRATION OF DRUGS ACTING ON POSTGANGLIONIC SYMPATHETIC NERVE FIBRES These drugs selectively block transmission in the postganglionic sympathetic nerve fibres and prevent the release of the transmitter substance at the termination of the nerve fibre. They do not antagonize the action of injected adrenaline on the effector organ. Guanethidine was given to four patients in doses of $30 \mathrm{mg}$. daily for a week. There was again no change in the effect of adrenaline on the tremor before and after the administration of this drug (Fig. 11).

\section{DISCUSSION}

These experiments have shown that adrenaline, and to a lesser extent isoprenaline, caused a marked increase in the amplitude of the tremor of Parkinsonism. This increase was clearly due to the pharmacological action of adrenaline, and not to general apprehension, since the injection of saline produced no effect. Noradrenaline, on the other hand, did not increase the amplitude or frequency of Parkinsonian tremor. This is in keeping with the findings of Barcroft, Peterson, and Schwab (1952), who observed that intravenous infusion of adrenaline caused an increase in tremor in $50 \%$ of their patients, whereas noradrenaline had no effect. The increase in the amplitude of the tremor in the present series was up to $400 \%$ and in frequency up to $30 \%$. These increases were remarkably constant; administration of the same amount of adrenaline to the same patients on different days had the same effects.

The site of action of the adrenaline was indicated by several experiments. First, the adrenaline administered intra-arterially had no effect on the tremor, whereas the same amount given intramuscularly produced a definite increase. This immediately suggested a central rather than a peripheral action. This suggestion was confirmed by more definitive experiments. The sympathetic ganglia were excluded as the site of action of the adrenaline by the absence of any change in its effect on tremor following the administration of ganglioplegic drugs (hexamethonium). Similarly, the postganglionic sympathetic fibre was eliminated by the administration of guanethidine which probably acts at this site. This likewise had no effect on adrenaline- induced tremor. The exclusion of a more peripheral action of adrenaline on effector cells was tried by the administration of phentolamine. No change in the adrenaline-produced tremor was observed after the administration of this agent, and therefore the action of adrenaline is clearly not at this site. In each of these experiments there was ample evidence that an adequate dose of the drug was being used, as the blood pressure was reduced in all the subjects even in the supine position.

The inhalation of isoprenaline in five subjects also produced an increase in tremor, but to a lesser degree than that produced by adrenaline. Tremor has in fact been reported as a side effect in the therapeutic use of isoprenaline (Gay and Long, 1949). The action of this catechol amine resembles more closely the action of adrenaline than of noradrenaline. It is present in very small amounts in the adrenal cortex and has a stimulant action on the central nervous system (Goodman and Gilman, 1955; Laurence and Moulton, 1960), hence may be affecting the same mechanism as does adrenaline.

Since the above experiments suggest no peripheral action of adrenaline, we must postulate a central action. The question arises as to its site, and why noradrenaline has no similar effect. There is considerable experimental evidence that adrenaline acts upon the brain-stem and particularly on the mesencephalic reticular formation. Bonvallet, Dell, and Hiebel (1954) showed that fluctuations in the electrical activity of the cortex paralleled those in sympathetic tone, and that adrenaline produced cortical activity, an effect which was abolished by intercollicular section of the brain-stem. They suggested, therefore, that circulating adrenaline activates the cortex through the postmesencephalic reticular formation. Rothballer (1956) also found that adrenaline in doses of 2 to $8 \mu \mathrm{g}$. $/ \mathrm{kg}$. produced alternating phases of activation and deactivation in the E.E.G. of unanaesthetized cats, and that coagulation of the junction of the mesencephalon and diencephalon abolished this response. They observed similar effects with noradrenaline, however. De Maar and Martin (1956) also observed E.E.G. activation with adrenaline but in contrast to Rothballer, they failed to secure any response from noradrenaline. Rothballer (1957) also observed that the local injection of adrenaline into the mid-brain reticular formation by a stereotactic method activated the E.E.G., an effect which was not obtained by injection into other sites.

Dell, Bonvallet, and Hugelin (1954) observed facilitation of spinal reflexes following intravenous adrenaline, an effect which was abolished by a retromamillary section of the brain-stem. It seems clear that adrenaline acts on the brain-stem reticular 
formation to produce effects in both the ascending and descending projections of this formation.

Electrical stimulation of the reticular formation in monkeys produces movements analogous to tremor (Jenkner and Ward, 1953). It is, therefore, possible that the aggravation in Parkinsonian tremor after the injection of adrenaline is due to the effect of this amine on the brain-stem reticular formation.

The increase of the tremor in Parkinsonism under emotional stress could be explained by this action of adrenaline on the reticular formation. The source of the adrenaline is uncertain. The suggestion that it comes from the sympathin, which is stored in the hypothalamus, seems unlikely, as in dogs only $14 \%$ of the sympathin is adrenaline and in cats only $7 \%$ (Vogt, 1954), the rest being noradrenaline which does not affect tremor. On the other hand, of the total catechol amines produced by the suprarenal glands, adrenaline constitutes $80 \%$ (Schacpdryver, 1961). Hence one may reasonably postulate that the increase in tremor in Parkinsonism during emotional stress is due to the secretion of adrenaline from the suprarenals.

The marked difference in the effects of adrenaline and noradrenaline remains unexplained. In these experiments adrenaline was given intramuscularly in doses of $1 \mathrm{mg}$., whereas similar concentrations of noradrenaline by this route may be dangerous. On the other hand, adrenaline and noradrenaline were given to three subjects by intravenous infusion in the same dosage without the noradrenaline producing any effect.

Noradrenaline produces a greater elevation of blood pressure than does adrenaline. It also causes bradycardia, which is an inhibitory phenomenon mediated through the baroceptors (Goodman and Gilman, 1955). It is possible that the inhibitory effects of noradrenaline through the baroceptors might cancel any direct stimulatory effects noradrenaline might have on the reticular formation. On the other hand, Rothballer (1959) has postulated central adrenoceptive mechanisms far more sensitive to adrenaline than to noradrenaline.

If then we postulate that adrenaline aggravates the tremor of Parkinsonism by acting upon the brainstem reticular formation, we must also postulate more than one tremor-producing mechanism in the brain-stem, for Domer and Feldberg (1960) have shown that, at least in cats, catechol amines inhibit other types of tremor. They found that the tremor produced by chlorpromazine and by pentobarbitone sodium was abolished by adrenaline administered into the lateral ventricle, and especially when the adrenaline was perfused through the aqueduct so as to bring it close to the brain-stem. Noradrenaline had a similar, though less powerful, effect. This is in striking contrast to the effects of intravenous administration in patients with Parkinsonism, for, as the present study has shown, though apparently acting centrally, adrenaline aggravates this type of tremor. Moreover, the action of adrenaline and noradrenaline is not uniform upon these tremor mechanisms, for noradrenaline has no effect upon Parkinsonian tremor.

In conclusion, it seems that the tremor of Parkinsonism is specifically aggravated by adrenaline, and to a lesser extent by isoprenaline, but not by the third commonly occurring catechol amine, noradrenaline. The most probable site of action of the adrenaline is on the reticular formation of the brainstem but the adrenaline must be acting at a specific site for other types of tremor appear to be inhibited by adrenaline acting upon the brain-stem.

\section{SUMMARY}

The action of adrenaline, isoprenaline, and noradrenaline on Parkinsonian tremor was studied in 25 patients. Adrenaline and to a lesser extent, isoprenaline, produced a definite and marked increase in the amplitude of the tremor. Also the frequency of the tremor was increased in $30 \%$ of the patients. Noradrenaline, on the other hand, had no effect.

An attempt was made to try to find the site of action of adrenaline by the administration of pharmacological agents. The experiments showed no evidence of peripheral action of adrenaline and a central action is postulated. The possible mechanism of the central action of adrenaline is discussed.

I wish to thank Dr. John Marshall under whose direction the work was carried out. The apparatus was provided by a grant from the Central Research Fund of the University of London.

\section{REFERENCES}

Barcroft, H., Peterson, E., and Schwab, R. S. (1952). Neurology, 2 , 154

Bonvallet, M., Dell, P., and Hiebel, G. (1954). Electroenceph. clin. Neurophysiol., 6, 119.

Dell, P., Bonvallet, M., and Hugelin, A. (1954). Ibid., 6, 599

De Maar, E. W. J., and Martin, W. R. (1956). Fed. Proc., 15, 416.

Domer, F. R., and Feldberg, W. (1960). Brit. J. Pharmacol., 15, 578.

Gay, L. N., and Long, J. W. (1949). J. Amer. med. Ass., 139, 452.

Goodman, L. S., and Gilman, A. (1955). The Pharmacological Basis of Therapeutics, 2nd ed. Macmillan, New York.

Jenkner, F. L., and Ward, A. (1953). A.M.A. Arch. Neurol. Psychiat., 70,489

Laurence, D. R., and Moulton, R. (1960). Clinical Pharmacology. Churchill, London.

Marazzi, A. G. (1960). In Inhibition in the Nervous System and Gammaaminobutyric Acid: Proc. int. Symposium, 1959. Pergamon Press, Oxford.

McLennan, H. (1961), J. Physiol (Lond), 158, 411.

Marshall, J., and Walsh, E. G. (1956). J. Neurol. Psychiat., 19, 260.

Rothballer, A. B. (1956). Electroenceph. clin. Neurophysiol., 8, 603.

(1957). Anat. Rec., 127, 359.

- (1959). Pharmacol. Rev., 11, 494.

Schacpdryver, A. F. (1961). J.S.C. med. Ass., 57, 45.

Vogt, M. (1954). J. Physiol. (Lond.), 123, 451. 